\title{
Unexplained Graft Dysfunction after Heart Transplantation-Role of Novel Molecular Expression Test Score and QTc-Interval: A Case Report
}

\author{
Khurram Shahzad, Martin Cadeiras, Kotaro Arai, Dmitry Abramov, Elizabeth Burke, \\ and Mario C. Deng
}

College of Physicians and Surgeons, Columbia University, New York, NY 10032, USA

Correspondence should be addressed to Khurram Shahzad, khurramshahzadmd@gmail.com and

Mario C. Deng, md785@columbia.edu

Received 20 March 2010; Accepted 13 May 2010

Academic Editor: Firat Duru

Copyright ( $\odot 2010$ Khurram Shahzad et al. This is an open access article distributed under the Creative Commons Attribution License, which permits unrestricted use, distribution, and reproduction in any medium, provided the original work is properly cited.

\begin{abstract}
In the current era of immunosuppressive medications there is increased observed incidence of graft dysfunction in the absence of known histological criteria of rejection after heart transplantation. A noninvasive molecular expression diagnostic test was developed and validated to rule out histological acute cellular rejection. In this paper we present for the first time, longitudinal pattern of changes in this novel diagnostic test score along with QTc-interval in a patient who was admitted with unexplained graft dysfunction. Patient presented with graft failure with negative findings on all known criteria of rejection including acute cellular rejection, antibody mediated rejection and cardiac allograft vasculopathy. The molecular expression test score showed gradual increase and QTc-interval showed gradual prolongation with the gradual decline in graft function. This paper exemplifies that in patients presenting with unexplained graft dysfunction, GEP test score and QTc-interval correlate with the changes in the graft function.
\end{abstract}

\section{Introduction}

Cardiac allograft rejection after heart transplantation (HTx) can involve both cellular and antibody mediated immune injury to the allograft. Hyperacute rejection is an antibodymediated process which occurs typically minutes to hours after transplantation. Acute cellular rejection (ACR) is a T-lymphocyte mediated process that occurs from the first week to years after HTx and is the major cause of graft loss early after HTx although its incidence declines after first year [1]. ACR is diagnosed by endomyocardial biopsy (EMB) and graded by degree of lymphocyte infiltration into myocardium according to International Society of Heart and Lung Transplantation (ISHLT) guidelines [2, 3]. Acute antibody mediated rejection (AMR) was reported to be present in $2 \%$ to $20 \%$ of EMBs after heart transplantation $[1,4-8]$. It is classically demonstrated by linear deposition of immunoglobulins and complement splitting products such as C4d in the vascular endothelium [8]. Cardiac allograft vasculopathy $(\mathrm{CAV})$ is one of the major causes of late graft dysfunction (GD). It is diagnosed by annual coronary angiography or intravascular ultrasound (IVUS). Historically invasive EMB was only indicated based on the clinical suspicion of rejection assessed by electrocardiographic and hemodynamic parameters. Recently, using postgenome-era high-throughput transcriptomic technology, a novel noninvasive molecular expression diagnostic test was developed and validated against EMB based criteria of ACR [9]. This peripheral blood mononuclear cell gene expression profiling (GEP) test with a score $<34$ has very high negative predictive value of $99.6 \%$ to rule out moderate/severe $(\geq 2 \mathrm{R} / 3 \mathrm{~A})$ ACR [10]. With the advancement in the immunosuppressive medications in the transplantation medicine, there is increased observed incidence of unexplained graft dysfunction in HTx patients. In this paper we present for the first time the role of longitudinal patterns of this novel molecular expression test and QTc-interval in a patient admitted with unexplained graft dysfunction. 


\section{Case Presentation}

A 59-year-old gentleman with a history of ischemic cardiomyopathy underwent orthotopic heart transplantation in our center on October 10, 2005. The donor was a 36-yearold male matched for body size with negative serologies for Cytomegalovirus and Toxoplasma. Postoperatively, his overall course was stable and followed with EMB without significant history of ACR. During the first 6 months rejection monitoring was EMB based. After the 9th month, we transitioned to non-invasive GEP test based monitoring [9] on the basis of consensus recommendations [10]. In the overlap phase (concomitant invasive and non-invasive monitoring), an EMB on June 6, 2006, showed absence of rejection (Quilty lesions) with a GEP test score of 26, right atrial pressure of $5 \mathrm{mmHg}$, pulmonary artery pressures of $31 \mathrm{mmHg}$ systolic, $11 \mathrm{mmHg}$ diastolic, and a mean of 22, pulmonary capillary wedge pressure of 13 , an $\mathrm{O}_{2}$ mixed venous saturation of $75 \%$, and a cardiac output (CO) by thermodilution of $5.861 / \mathrm{min}$ (Cardiac Index 2.551/min/sqm). A follow up biopsy on January 11, 2007 showed ISHLT grade 0 with a GEP score of 28 (Table 1). From that time on, the patient was followed non-invasively with the GEP test and echocardiography and had a clinically stable course until January 2008. A follow up echocardiogram obtained on January 17, 2008 showed reduction in left ventricular ejection fraction (LVEF) from normal to $43 \%$. The patient's immunosuppressive regimen at that moment included cyclosporine (CsA) $100 \mathrm{mg}$ twice a day, mycophenolate $250 \mathrm{mg}$ twice a day and prednisone $5 \mathrm{mg}$ daily. In addition, the patient was also receiving pravastatin $20 \mathrm{mg}$, aspirin $81 \mathrm{mg}$, doxazosin $1 \mathrm{mg}$, amlodipine $2.5 \mathrm{mg}$ and carvedilol $25 \mathrm{mg}$.

On January 29 2008, the patient presented to our clinic with atypical chest pain, shortness of breath, and hemoptysis. His Initial evaluation revealed blood pressure of $120 / 80 \mathrm{mmHg}$, heart rate $92 \mathrm{bpm}$ regular, temperature 98.7, and respiratory rate of 22. His NYHA functional class was III-IV, his lung auscultation was remarkable for bilateral crackles, and his heart auscultation was positive for an S3 gallop. The abdomen was soft without organomegaly and the patient had no lower extremity edema. A chest Xray showed new extensive right perihilar disease. An EKG showed no acute changes but his QTc-interval duration was $530 \mathrm{~ms}$. Right heart catheterization and an EMB were done emergently. Hemodynamics showed Pulmonary Artery (S/D/M): 62/33/45, Pulmonary Capillary Wedge $(\mathrm{a} / \mathrm{v} / \mathrm{M})$ : $34 / 47 / 39$, Right Atrium (a/v/M): 16/14/13, a Mixed Venous $\mathrm{O}_{2}$ Saturation of $51 \%$ and $\mathrm{CO}$ by thermodilution of $3.20 \mathrm{l} / \mathrm{min}$ (Cardiac Index $=1.38 \mathrm{l} / \mathrm{min} / \mathrm{sqm})$.

The review of the patient posttransplant management during the last year showed a progressive declination of the graft function within the range of normality values (Table 1), prolongation of the QTc-interval on the EKG, and progressive increase in his GEP scores (Figure 1). After the clinically indicated $\mathrm{EMB}$, patient was treated with intravenous steroids under the presumed diagnosis of ACR. The EMB showed focal, mild (1A/1R) ACR with nodular endocardial infiltrate (Quilty effect). A C4d immunohistochemical staining was negative for AMR. Respiratory viral panels were negative,

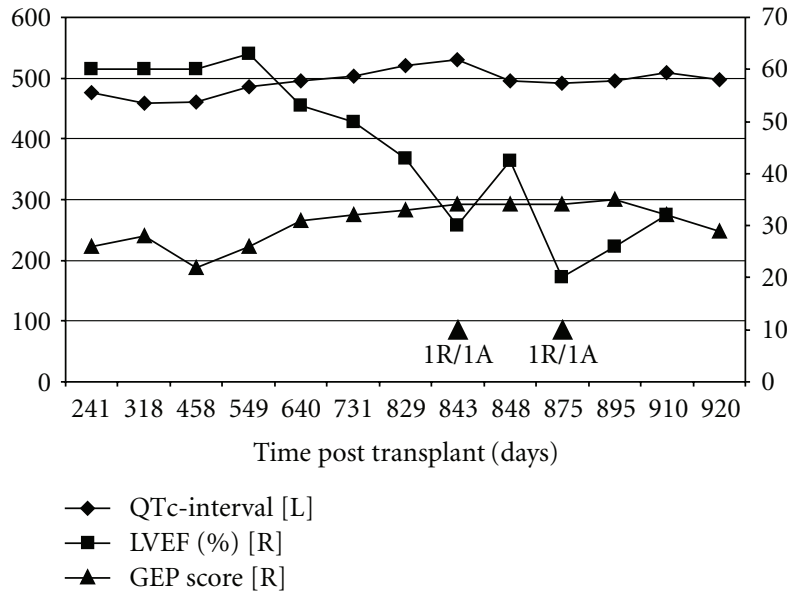

FIGURE 1: Longitudinal post-HTx changes in the GEP test score, QTc-interval, and Left Ventricular Ejection Fraction. L = left axis; $\mathrm{R}=$ right axis; $\mathrm{LVEF}=$ left ventricular ejection fraction; GEP = gene expression profiling test score; QTc $=$ corrected $\mathrm{QT}$ interval.

and viral inclusions or associated inflammatory response was not found on the EMB. The patient was treated with intravenous (pulse) methyl-prednisolone for three consecutive days ( 500 milligrams per day) followed by an oral taper of methyl-prednisone in addition to diuretics. A coronary angiogram done on February 05, 2008 showed mild pruning of the distal coronary arteries. A follow-up echocardiogram 4 days after completing treatment showed improvement in regional wall motion abnormality and a significant increase in the LVEF to $40 \%-45 \%$. The patient was discharged home in a stable condition. On 03/04/2008, a follow-up $\mathrm{EMB}$ and right heart catheterization were obtained which showed impaired graft function and 1A/1R biopsy grade with negative C4d staining. The patient was treated with intravenous methyl-prednisolone $(500 \mathrm{mg})$ and a steroid taper. New evaluation with EMB on 04/07/2008 showed absence of lymphocytic infiltrates or C4d deposition. Subsequent evaluation of the graft function by echocardiography showed persistent allograft dysfunction (Table 1).

\section{Discussion}

There were no findings consistent with ACR and AMR in our case. CAV is present in about half of all HTx recipients at 5 years posttransplantation. Although our patient had evidence of mild pruning of the distal coronary vessels on the angiography but there was no significant blockage of any of the main vessels. These angiographic findings were unable to explain the degree of graft dysfunction.

Infectious etiologies are a potential cause for primary or secondary allograft dysfunction during the first years after transplantation with cytomegalovirus as the most common etiologic agent [11]. Repetitive blood cultures and PCR analyses and the absence of intranuclear inclusions in the endomyocardial samples made this possibility very unlikely. Considering the initial presentation of the patient with acute respiratory infiltrates would raise the consideration of a 


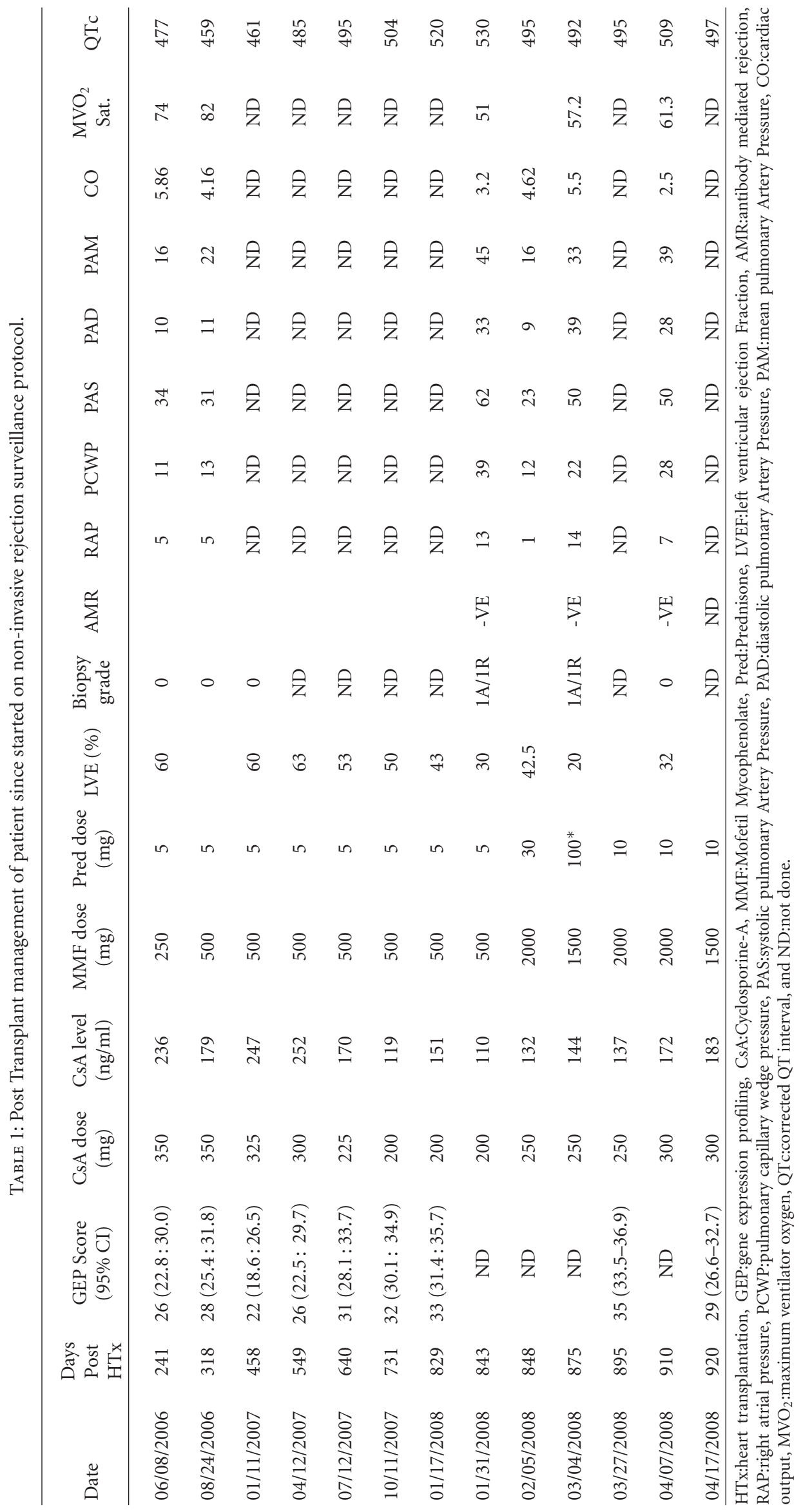


severe systemic acute infection, potentially of viral origin leading to graft loss which has been increasingly reported [12]. An infiltrative myocardial disease of the recipient could be suspected such as amyloidosis or lymphoproliferative disease affecting the heart. Post-HTx lymphoproliferative disease has been reported to have tropism for the transplanted organs and to be a cause of cardiac allograft failure [13]. Neither the clinical findings during this admission nor the biopsy raised this suspicion.

In the present case, the review of his medical history showed progressive declination of the graft function within the range of normality, with partial improvement after steroid treatment that suggests an acute inflammatory alloimmune mechanism with a negative biopsy. EMB has been used for the last 35 years to screen patients for ACR although it has been limited by invasiveness, complexity, discomfort, complication-proneness, interpretation variability and late detection of rejection. In the current era and state of practice in HTx, the limitations of EMB have been recalled. The likelihood of consensus for the diagnosis of ACR in eyes of highly experienced pathologist is less than expected with $78 \%$ best agreement rate for $3 \mathrm{~A} / 2 \mathrm{R}$ rejection and significantly lower for grades $1 \mathrm{~B} / 1 \mathrm{R}, 1 \mathrm{~A} / 1 \mathrm{R}$, and $2 / 1 \mathrm{R}$. There is $50 \%$ downgrading of rejection grade when an independent local pathologist reading is reinterpreted by a core of expert pathologists [14].

In original CARGO study, the cutoff of 34 on the GEP test score scale was selected to adjust for the high negative predictive value (NPV) of the test with the intention to rule out ACR. In our patient, GEP test score mostly remained in the lower 30s range. This could be explained by the absence of documented evidence of moderate/severe ACR as defined by histology ( $\geq 2 \mathrm{R} / 3 \mathrm{~A})$ against which GEP test was developed and validated. Most recently, we have analyzed the variability of the molecular score within and between patients suggesting that not only a cutoff value is important but the time dependent variability of the molecular score may provide a more personalized evaluation [15]. Furthermore, data from CARGO study showed that independently from the cut-off value set for the purpose of the study, as the agreement of rejection diagnosis increased among pathologists, also did the molecular score [9].

In the post implementation clinical experience of GEP test we have demonstrated the relationship of the GEP test score with echocardiographic and electrocardiographic parameters of allograft rejection [16]. In the present case, longitudinal evaluation of the results of the GEP test suggested a progressive increase in the molecular score from a deep quiescent range to an alloactivated status. The time dependent and sustained trend of the molecular test showed a significant variability that followed the progressive left ventricular function deterioration, prolongation of the QTcinterval, and development of acute decompensated graft failure that lead to hospitalization. The presentation of GD without histological evidence of significant ACR, absence of C4d deposition, and other surrogates of rejection suggest the limitation of currently available diagnostic tools. With the failure of currently known histopathological mechanisms to explain the involved immune mechanisms there is need to develop accurate methods beyond histological criteria to detect/predict patients with unexplained graft dysfunction.

\section{Conclusion}

This case report exemplifies that in patients with unexplained graft dysfunction, GEP test score and QTc-interval correlate with the changes in the graft function. These patients are empirically treated as rejection or even infection without an objective proof to support the diagnosis or treatment for these severe life threatening conditions. Furthermore, we use medical treatments that may lead to severe adverse events without exactly knowing which the entity that we are treating is. Further studies are required to better understand the immune mechanisms of unexplained GD and to develop accurate diagnostic tools beyond histological criteria for appropriate treatment interventions.

\section{Abbreviations}

$\begin{array}{ll}\text { HTx: } & \text { Heart transplantation } \\ \text { GEP: } & \text { Gene expression profiling } \\ \text { NYHA: } & \text { New York Heart association } \\ \text { ISHLT: } & \text { International Society of Heart and Lung } \\ & \text { Transplantation } \\ \text { QTc: } & \text { Corrected QT interval } \\ \text { EKG: } & \text { Electro cardiogram } \\ \text { CsA: } & \text { Cyclosporine } \\ \text { CO: } & \text { Cardiac output } \\ \text { HLA: } & \text { Human leukocyte antigen } \\ \text { PCR: } & \text { Polymerase chain reaction. }\end{array}$

\section{Authors' Contributions}

KS collected and analyzed the biopsy, hemodynamic and drug dose and level information; wrote the manuscript and built it in its current form for submission. MC collected the clinical data regarding the different forms of rejection e.g. acute cellar rejection, antibody mediated rejection and chronic rejection. He wrote the initial draft and compiled the references. KA did the echocardiograms for the patient and collected the echocardiographic data. DA was the resident taking care of the patient during hospital stay. EB provided the donor related information. MCD is the creator of the GEP test and was responsible for the overall interpretation of the results. All authors read and approved the final manuscript.

\section{Acknowledgment}

K. Shahzad and M. Cadeirast contributed equally to this work.

\section{References}

[1] S. Subherwal, J. A. Kobashigawa, G. Cogert, J. Patel, M. Espejo, and B. Oeser, "Incidence of acute cellular rejection and noncellular rejection in cardiac transplantation," Transplantation Proceedings, vol. 36, no. 10, pp. 3171-3172, 2004. 
[2] M. E. Billingham, N. R. B. Cary, M. E. Hammond, et al., "A working formulation for the standardization of nomenclature in the diagnosis of heart and lung rejection: heart rejection study group," Journal of Heart Transplantation, vol. 9, no. 6, pp. 587-593, 1990.

[3] S. Stewart, G. L. Winters, M. C. Fishbein, et al., "Revision of the 1990 working formulation for the standardization of nomenclature in the diagnosis of heart rejection," Journal of Heart and Lung Transplantation, vol. 24, no. 11, pp. 17101720, 2005.

[4] E. H. Hammond, R. L. Yowell, S. Nunoda, et al., "Vascular (humoral) rejection in heart transplantation: pathologic observations and clinical implications," Journal of Heart Transplantation, vol. 8, no. 6, pp. 430-443, 1989.

[5] E. A. Rose, C. R. Smith, G. A. Petrossian, M. L. Barr, and K. Reemtsma, "Humoral immune responses after cardiac transplantation: correlation with fatal rejection and graft atherosclerosis," Surgery, vol. 106, no. 2, pp. 203-208, 1989.

[6] R. Cherry, H. Nielsen, E. Reed, K. Reemtsma, N. Suciu-Foca, and C. C. Marboe, "Vascular (humoral) rejection in human cardiac allograft biopsies: relation to circulating anti-HLA antibodies," Journal of Heart and Lung Transplantation, vol. 11, no. 1, part 1, pp. 24-30, 1992.

[7] L. W. Miller, A. Wesp, S. H. Jennison, et al., "Vascular rejection in heart transplant recipients," Journal of Heart and Lung Transplantation, vol. 12, no. 2, pp. S147-S152, 1993.

[8] M. A. Lones, L. S. C. Czer, A. Trento, D. Harasty, J. M. Miller, and M. C. Fishbein, "Clinical-pathologic features of humoral rejection in cardiac allografts: a study in 81 consecutive patients," Journal of Heart and Lung Transplantation, vol. 14, no. 1, part 1, pp. 151-162, 1995.

[9] M. C. Deng, H. J. Eisen, M. R. Mehra, et al., "Noninvasive discrimination of rejection in cardiac allograft recipients using gene expression profiling," American Journal of Transplantation, vol. 6, no. 1, pp. 150-160, 2006.

[10] R. C. Starling, M. Pham, H. Valantine, et al., "Molecular testing in the management of cardiac transplant recipients: initial clinical experience," Journal of Heart and Lung Transplantation, vol. 25, no. 12, pp. 1389-1395, 2006.

[11] T. A. Gonwa, J. E. Capehart, J. W. Pilcher, and P. A. Alivizatos, "Cytomegalovirus myocarditis as a cause of cardiac dysfunction in a heart transplant recipient," Transplantation, vol. 47, no. 1, pp. 197-199, 1989.

[12] D. Ivan, O. H. Frazier, and J. Abrams, "Fatal disseminated adenoviral infection in an adult heart transplant patient," Journal of Heart and Lung Transplantation, vol. 23, no. 10, pp. 1209-1212, 2004.

[13] H. J. Eisen, D. Hicks, J. A. Kant, et al., "Diagnosis of posttransplantation lymphoproliferative disorder by endomyocardial biopsy in a cardiac allograft recipient," Journal of Heart and Lung Transplantation, vol. 13, no. 2, pp. 241-245, 1994.

[14] C. C. Marboe, M. Billingham, H. Eisen, et al., "Nodular endocardial infiltrates (Quilty lesions) cause significant variability in diagnosis of ISHLT Grade 2 and $3 \mathrm{~A}$ rejection in cardiac allograft recipients," Journal of Heart and Lung Transplantation, vol. 24, no. 7, pp. S219-S226, 2005.

[15] M. C. Deng, et al., "Low variability of intraindividual longitudinal leukocyte gene expression profiling cardiac allograft rejection scores," Journal of Transplantation. In press.

[16] K. Shahzad, J. He, Q. Li, Q. A. Aziz, and M. C. Deng, "Relationship between prolonged QTc interval, cardiac allograft dysfunction and elevated molecular gene expression profiling test score after heart transplantation," Journal of Heart and Lung Transplantation, vol. 29, no. 6, pp. 711-713, 2010. 


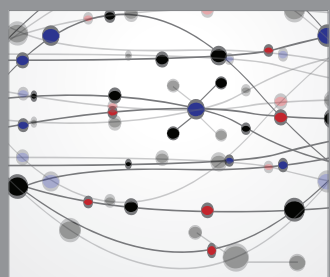

The Scientific World Journal
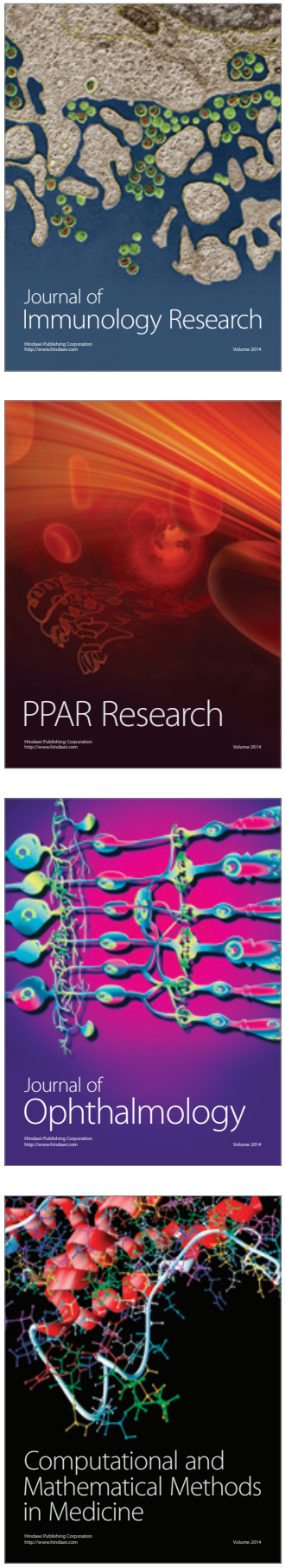

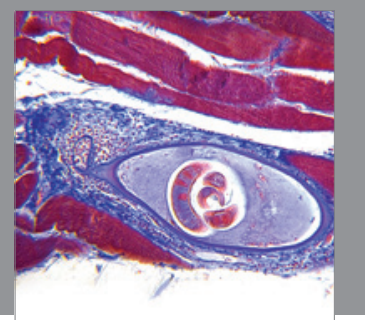

Gastroenterology

Research and Practice
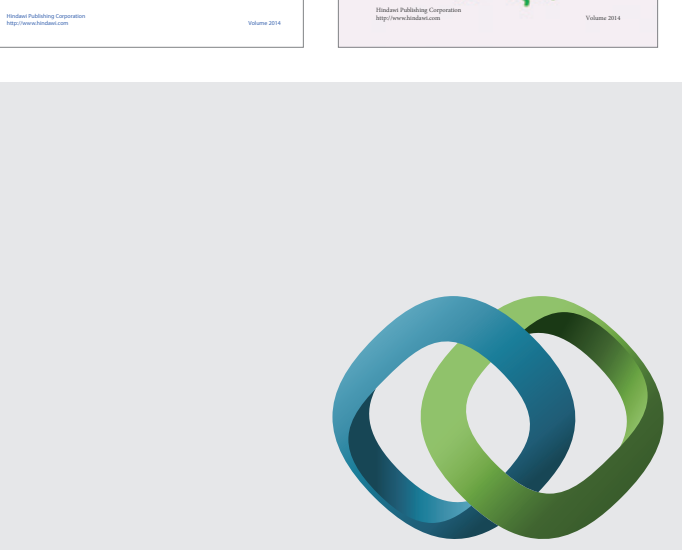

\section{Hindawi}

Submit your manuscripts at

http://www.hindawi.com
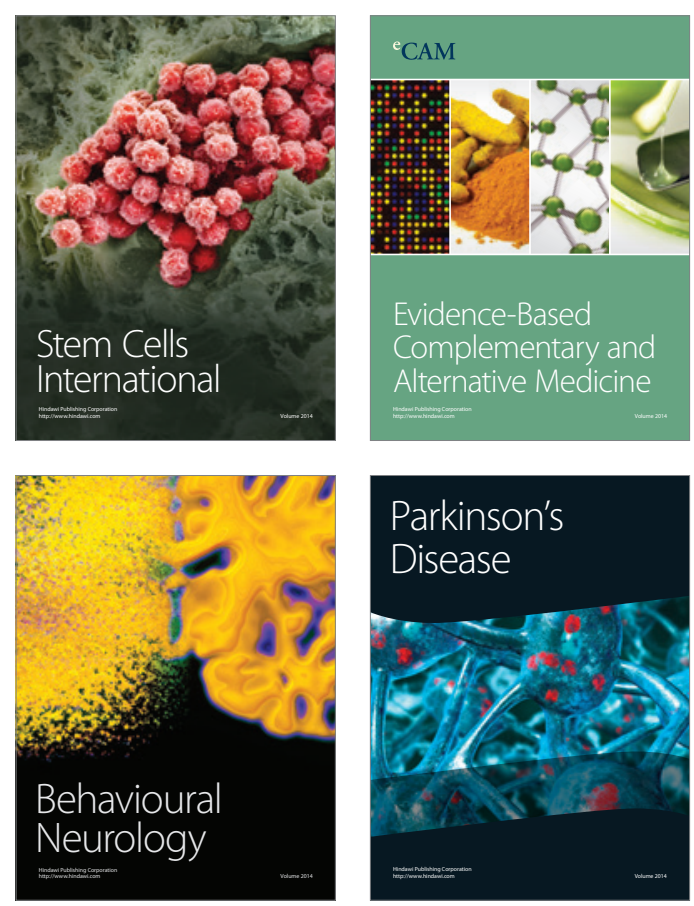

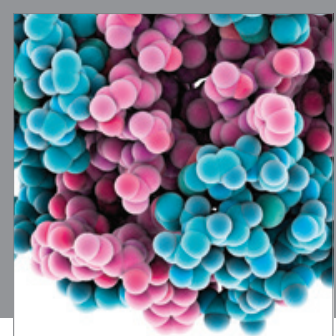

Journal of
Diabetes Research

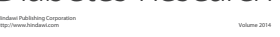

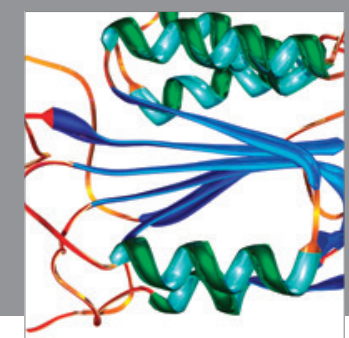

Disease Markers
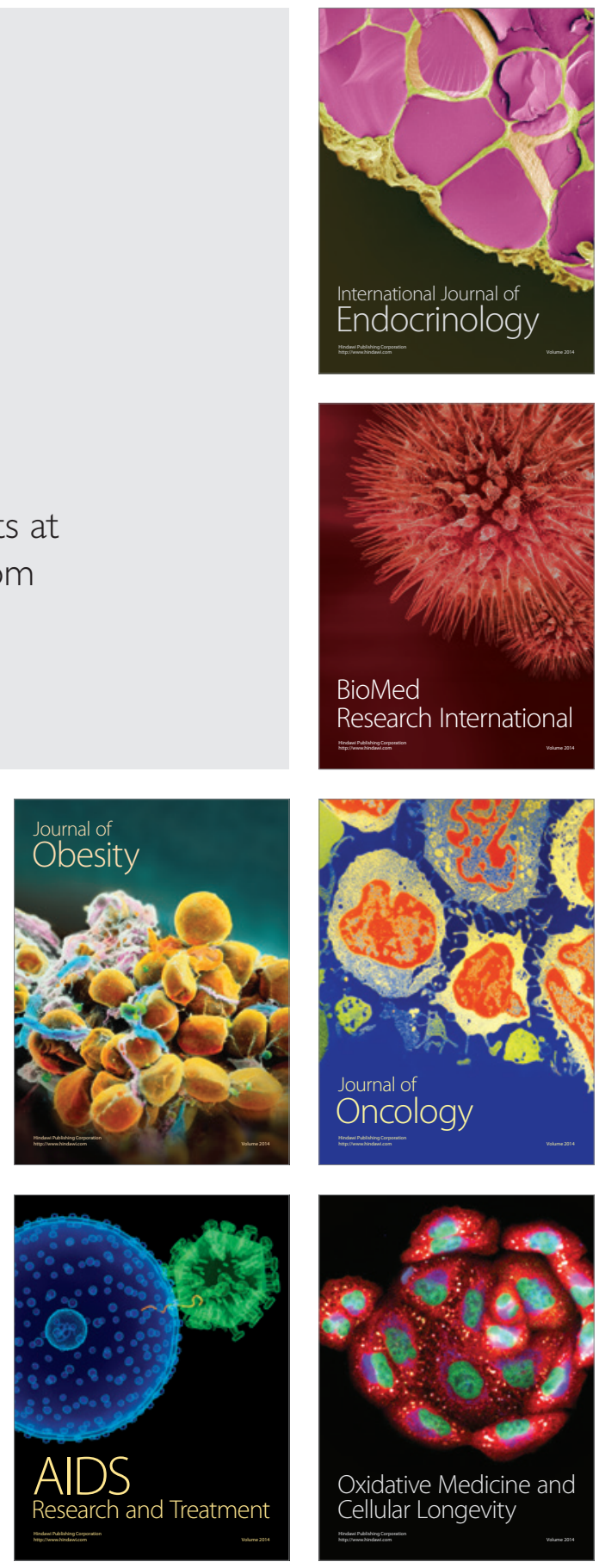